\title{
Reaction-Diffusion Manifolds and Global Quasi-linearization: Two Com- plementary Methods for Mechanism Reduction
}

\author{
V. Bykov ${ }^{1 * *}$ and U. Maas ${ }^{1}$ \\ ${ }^{1}$ Institute of Technical Thermodynamics, Karlsruhe Institute of Technology (KIT), Germany
}

\begin{abstract}
The paper outlines the current state in the model reduction of systems governing reacting flows by manifold methods. The main idea of such approaches is based on the fact that any reduced model defines a manifold of low dimension imbedded in the system composition/state space. In this respect the decomposition into relatively fast and slow motions due to multiple time scales present in the system is a crucial property of the reacting system. It allows the application of the geometrical framework of slow and fast invariant manifolds to model reduction. Recently developed approaches, namely, the so-called Reaction-Diffusion Manifolds (REDIMs) and Global-Quasi Linearization (GQL) are in the focus of this work. The methods extend and follow the well known ILDM method. The paper discusses both the theoretical basis of the approaches and detailed implementation schemes for studying, reducing and simulating the reacting flows systems. Simple yet containing all features of the reacting flows models of n-heptane/air and syngas/air systems are used to illustrate and verify the methods.
\end{abstract}

Keywords: Reacting flows, chemical kinetics, model reduction, ILDM, slow/fast manifolds, invariant manifolds.

\section{INTRODUCTION}

The main our goal of this work is to discuss resent progress in understanding, developing the general framework of model reduction and its implementation to systems governing reacting flows. The key idea of our approach connected to so-called low dimensional manifolds with definite properties that can be efficiently used for model reduction purposes.

The idea is not new, it appeared e.g. in $[1,2]$, where an efficient method of approximation of low-dimensional manifolds of so-called slow motions (Intrinsic Low-Dimensional Manifolds (ILDMs)) has been suggested. It has been proven that the method approaches the slow manifold with the accuracy of first order of magnitude with respect to the standard analysis by the Singularly Perturbed Systems (SPS) theory $[3,4]$. Many similar approaches based on the local analysis given by eigenvalues and invariant subspaces of the Jacobian matrix can be found in the literature (see [5-10] for more references and descriptions).

However, one important technical feature, namely, the so-called tabulation strategy proposed by the ILDM method for an explicit manifold representation, has made it as a robust and comprehensive tool of model reduction. It allows a fast and easy implementation of the reduced model to modeling and simulation of reacting flow systems. It is very convenient from numerical point of view and allows to save up to $\mathrm{O}\left(\mathrm{m}_{\mathrm{f}}^{2}\right)$ operations in the case $\mathrm{m}_{\mathrm{f}}$ dimensions have been reduced [12]. The application of tabulation strategy made possible strategies using particle methods (PDF approximation) to model the chemical reaction in the flow [11].

*Address correspondence to this author at the Institute of Technical Thermodynamics, Karlsruhe Institute of Technology (KIT), Germany; Tel: (+49) 721 6088746; Fax: (+49) 721 6083931; E-mail: bykov@ @itt.uni-karlsruhe.de
The basis of all mechanism reductions are starting mechanisms that are either fully detailed mechanisms with up to several hundreds species or so-called skeletal mechanisms where unimportant reactions or species have already been removed (see e.g. [13] for detail). How to chose the detailed/skeletal model, and to what extent it should describe phenomena depends merely on purposes of an investigation and cannot be decided generally. For a further reduction to very small reduced mechanisms either physicochemical knowledge is applied to identify species in steady states or reactions in partial equilibrium etc. or mathematical methods are used which identify slow rate limiting processes which have to be retained in the system and fast processes which are eliminated [2-5].

Accordingly, properties of a reacting system important for model reduction and the recent progress in understanding of the theoretical and practical aspects of model reduction are in the focus of the current work.

\subsection{Rigorous Mathematical Description}

Mathematically, a simple system describing reacting phenomena is a pure homogeneous system, which is presented by a system of ordinary differential equations (ODEs) that describes the chemical system evolution based on the mass action law. These reaction equations describe the evolution of the thermo-chemical state vector in linear vector space $\psi=\left(\psi_{1}, \ldots, \psi_{n}\right)^{T}$ in time, where $\psi_{j}$ represents such quantities as the pressure of the mixture $p$, the enthalpy $h$, the mass fraction/mole concentration of chemical species or their specific mole numbers $\psi_{i}=w_{i} / M_{i}, i=1, \ldots, n_{s}$ as it is used in the ILDM method. In vector notation the system of governing equations of a homogeneous system can be written in autonomous form as (see e.g. [14, 15]) 


$$
\frac{d \psi}{d t}=F(\psi), \quad \psi \in \Omega \subset R^{n} .
$$

Here the so-called chemical source term $F$ represents chemical kinetics mechanism with reaction constants given typically by the Arrhenius kinetic [16]. It describes the evolution of $\mathrm{n}_{\mathrm{s}}$ chemical species $\left(n=n_{s}+2\right.$, with two additional variables describing the thermodynamic properties) participating in $n_{r}$ elementary chemical reactions.

The main question of the system (1) reduction from a mathematical point of view can be understood as follows. A model reduction is a reformulation process of the system (1) in an appropriate form by introducing the reduced space $\theta=\left(\theta_{1}, \ldots, \theta_{m}\right), m<n$ consisting of new variable set, such that the solution of the system (1) will be accurately described by reduced model

$$
\frac{d \theta}{d t}=\tilde{F}(\theta), \quad \theta \in R^{m} .
$$

How the systems (1) and (2) relate to each other is a basic question of model reduction, i.e. the question of how the detailed $\psi=\left(\psi_{1}, \ldots, \psi_{n}\right)$ and reduced spaces $\theta=\left(\theta_{1}, \ldots, \theta_{m}\right)$ are connected has to be in the focus of any model reduction strategy. In principle, one needs to determine the relation between these spaces in order to reduce the system. In the case the reduced space can be accurately represented in an explicit form

$$
M=\left\{\psi=\psi(\theta), \quad \theta \in R^{m}\right\},
$$

by a low-dimensional manifold imbedded in the detailed system (1) state space. Then, the system (1) can be projected onto the manifold $M$ yielding the reduced form $\frac{d \theta}{d t}=\psi_{\theta}^{+}(\theta) F(\psi(\theta)) \equiv \tilde{F}(\theta), \quad \theta \in R^{m}$.

the Moore-Penrose pseudo inverse $\psi_{\theta}^{+}(\theta)$ [17] has been used in (4), which is a well defined function unless the tangent space $T M$ given by $\psi_{\theta}(\theta)$ degenerates. All conventional methods like, for instance, the Quasi Steady States Approximation (QSSA) method (see e.g. [18, 19]) follow this way implicitly, i.e. in order to access the reduced space or manifold (3) it is assumed that some species are in steady states. It gives an implicit function, as the RHS of (1) that equals to zero for the steady state species. This defines the reduced manifold. It is obvious, that in this case the problem of the choice of the steady states is not trivial and cannot be solved generally, because original coordinates or species might not be suitable for the assumption made.

Obviously, the reduction of a given arbitrary system with prescribed dimension and accuracy is almost always impossible in principle, but fortunately, systems governed by chemical reactions have certain properties that, nevertheless, make the model reduction feasible.

The most important is the existence of various scales or multi-scale phenomena. It is the well known feature of chemically reacting flows, both in experiments and in detailed model simulations it is observed that not the entire possible range of the thermo-chemical state space is typically accessed by the system states (solutions), but only a part of it [20].

Fig. (1) shows direct numerical simulation results for a turbulent non-premixed flow $(\operatorname{Re}=210)$ [20] that support the observation above. In this figure, it is possible to see that in spite of the very complex system behavior in the physical space $(x, y)$ (upper set); the whole range of the physical states is used to define the state space accessed by the system
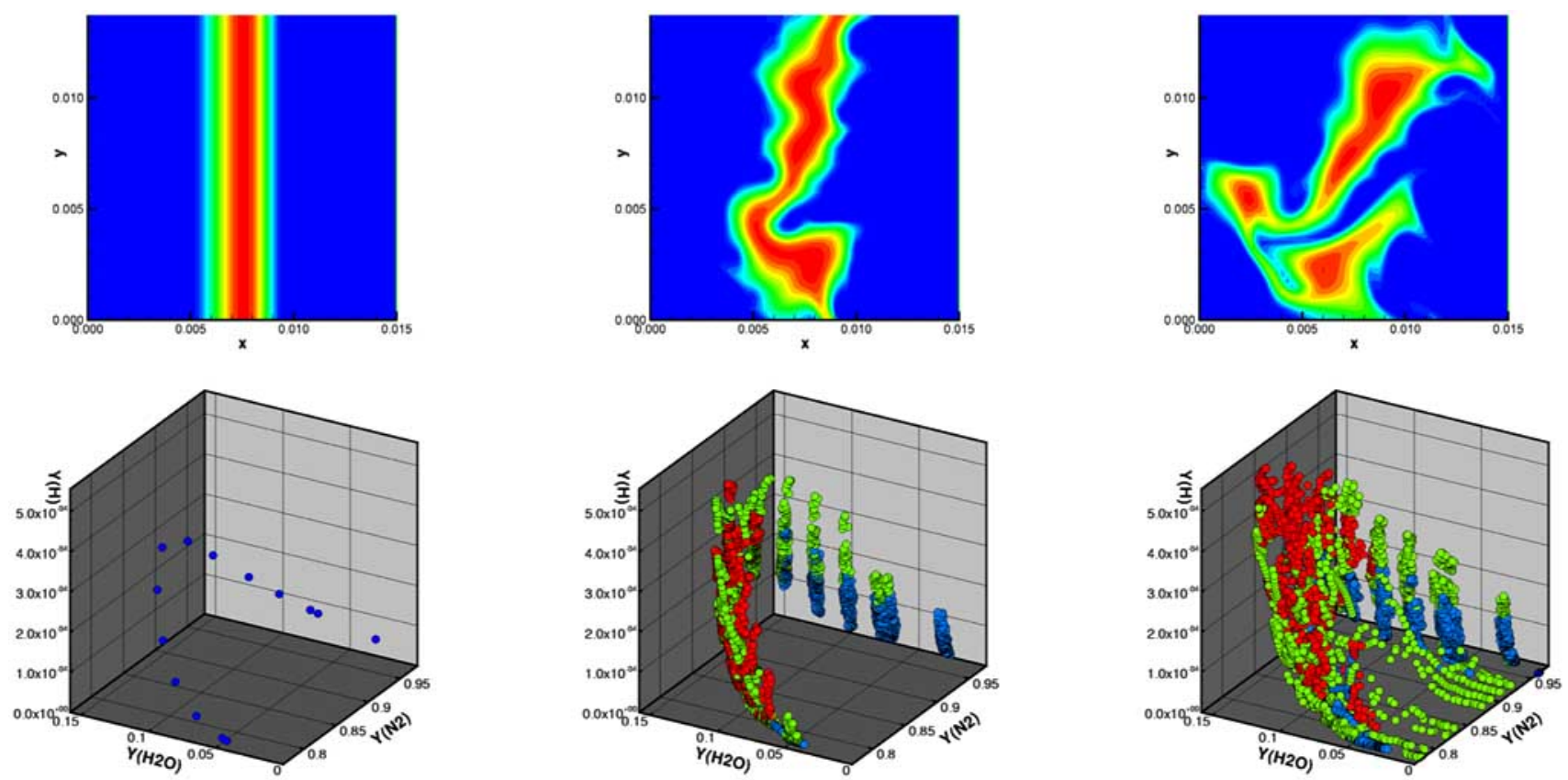

Fig. (1). DNS of a turbulent non-premixed hydrogen flame. Upper figures show temperature contour lines, lower figures present samples of system states in projection to corresponding mass fractions [20]. 
in projection to some mass fractions (bottom set) has a relatively simple and confined form. Therefore, when this part has a low-dimensional structure, it can be approximated efficiently by low-dimensional manifolds. These manifolds and their properties (existence, attractiveness, stability, and slowness/fastness) are very important and should be carefully studied before proceeding with model reduction.

An efficient and robust model reduction strategy should not only allow the estimation or approximation of the needed reduced space or manifold (the manifold containing the reduced system dynamics), but it has to provide with the tool for its analysis with respect to the properties above.

There is another astounding feature of the reduced spaces/manifolds concerning the combustion chemistry that can be efficiently used in applications. It is the hierarchy not only with respect to dimensions, but with respect to increasing complexity from lower to higher hydrocarbons. Fig. (2) shows particular solution trajectories for several high hydrocarbon systems in projection to common species. One sees that all trajectories follow almost same low dimensional structures, the system solution relax towards common lower dimensional manifolds. Although, models have significantly different dimensions (typically $\mathrm{H}_{2} \sim 8, \mathrm{H}_{2} / \mathrm{CO} \sim 13, \mathrm{CH}_{4} \sim$ 34 etc.) at final stages they all relax onto 2D ILDM, then join to one line yielding 1D manifold and finally enter the equilibrium point (red circle, see e.g. [21] for detailed investigations).

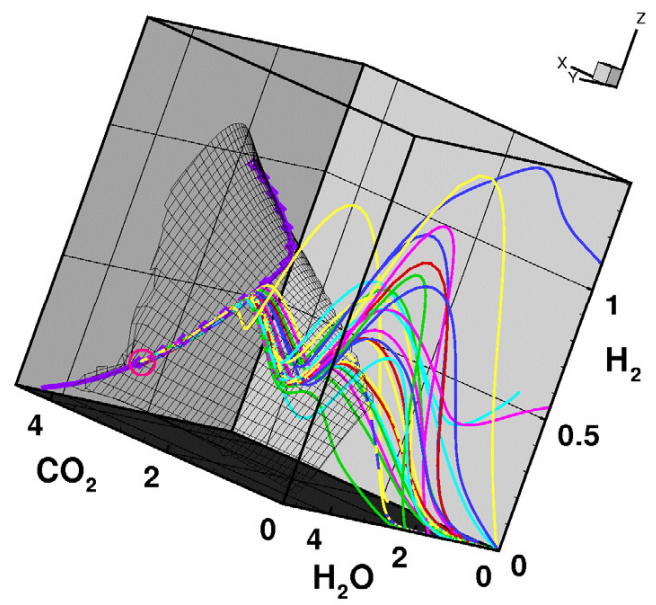

Fig. (2). The projection of detailed state spaces onto specific mole numbers of several high hydrocarbon systems with an imbedded 2D ILDM (black mesh) and detailed systems' trajectories [21] (colored lines).

In the next sections, theoretical basics of manifold based methods of mechanism analysis and model reduction are presented and discussed. Several variants of manifold methods exist [8], [22]. The philosophy behind these methods is the same but they differ both in the details of the concepts and in the methods of implementation.

\subsection{Multi-Scales Phenomena and Important Questions for Model Reduction}

Most of the existing reduction methods try to characterize the multi-scale hierarchical structure of the original system and then in different ways use this special feature for reduction purposes. In spite of many advantages of available currently automatic reduction methods there are principal, fundamental drawbacks of such approaches. The first is connected to the fact that an attractive property of the manifold approximating the reduced system dynamics does not exist everywhere in the whole domain of interest in the state space. It means that the system dynamics cannot be reduced by the slow manifold in some part or in some sub-domain of the whole domain of interest.

The second major drawback is a lack of a rigorous methodology for the global characterization of different scales by the so-called decomposition or separation into slow and fast processes. Most approaches have a local character only, i.e. sensitivity analysis on particular system trajectories, Jacobian matrix analysis etc., while their results are automatically extended to the whole domain. Fig. (3) illustrates a possible situation of loosing the stability of the slow manifold. The so-called turning manifolds - set of points, where fast directions of relaxation process tangential to the slow manifold (red dashed curves), represent a serious obstacle for precise model reduction. These objects normally separate the stable and unstable parts of slow manifolds. Obviously, an unstable part of the slow manifold cannot be used for a successful model reduction and local analysis is unable to provide with such information.

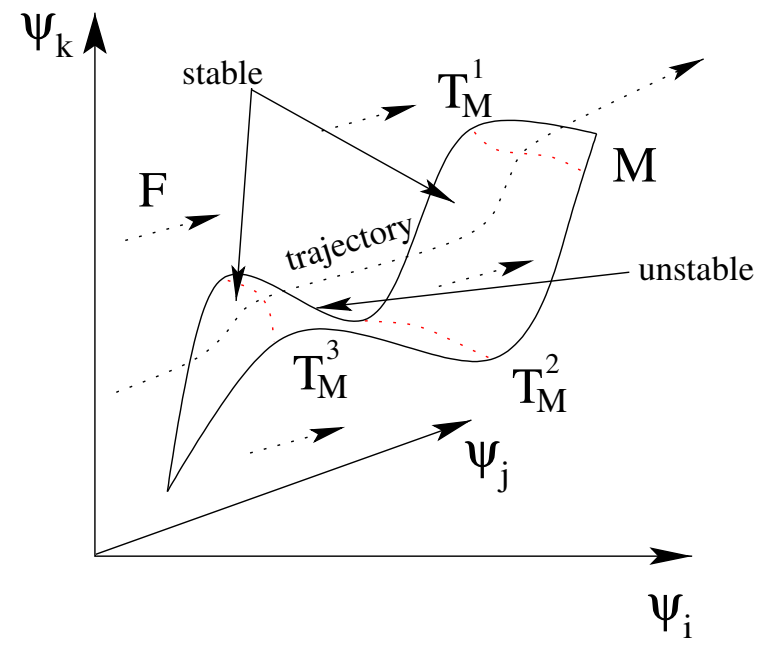

Fig. (3). Sketch of reduced state space $M$ as a manifold of slow motions, $\mathrm{F}$ shows direction of fast relaxation motions of the system and red curves $\mathrm{T}_{\mathrm{M}}^{\mathrm{i}}$ are turning manifolds separate the stable and unstable parts of $\mathrm{M}$.

For that reason, by now, there are a number of "tricks" which make the implementation of conventional reduction techniques possible. For instance, by implementing fake state space relations which are used often in the domain where the slow manifold does not exist, by tuning of reduced models etc.

The next difficult and fundamental problem of model reduction is the coupling of molecular transport with thermochemical processes. It is the very difficult problem normally solved by either neglecting such an interaction at all or by applying operator splitting methodologies (see e.g. [23]), which implies ignoring of the coupling locally either in time or in space. In these cases, the interaction and influence of the molecular transport is not taken into account and excluded from the reduced model. 
Obviously, an algorithm which overcomes these drawbacks and answers the question above in most general case has to be designed. It should provide with the tool of the system analysis of low-dimensional manifolds and their properties (important for model reduction) and allow treating systematically the coupling of the chemical and transport sub-processes.

Accordingly, any successful automatic reduction method should be able to answer or has to deal with the following important questions concerning discovery the multi-scales hierarchy of the system under consideration:

1. Are there multi-scales in the system (1)?

2. If yes, then how these scales can be used or decomposed?

3. What is an explicit form of the decomposition i.e. what the simplest representation of the system that allows to use multi-scales and handle model reduction in simple manner?

4. How to find out slow and fast manifolds (as directions of fast relaxations) and define their properties (i.e. for the slow manifold: existence, stability, attractiveness, basin of attraction etc.)?

5. Is it possible to single out limits of application of particular asymptotic behavior, i.e. how to handle transient behaviors?

6. How can the reduced model include information about coupling with the transport properties of the reacting flow?

\subsection{Implementation Aspects of Model Reduction}

As for the implementation of a manifold-based reduction scheme, it must contain following stages. The first stage is to find a low dimensional manifold in the state space that can be applied to approximate the full system dynamics in terms of local/inner coordinates of the manifold $\theta$ as it is described by (1-4). The second stage is the analysis of the overall dynamics on the manifold and its properties like existence, slowness, attractiveness, boundaries, optimal dimensions etc. The third is the actual model reduction with manifold representation (by defining inner coordinates, tabulation, improvement of manifold representation etc.) and projection of the system of governing equations and its initial conditions on the "constructed" manifold, i.e. the transformation to the new coordinates $\theta$.

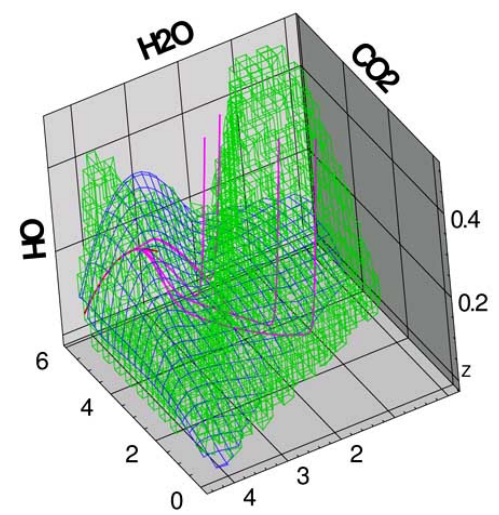

Therefore, in order to design a sophisticated tool of model reduction answering automatically the main questions above, two complementary subjects should be handled, one connected to theoretical aspects and another is purely practical, namely,

(i) The model reduction should be considered in the most general formulation by finding a suitable framework and formulating necessary notations and helpful constructions.

(ii) When the basic concepts of model reduction are well understood and studied, the most efficient way of application has to be found.

Each currently used method has its advantages and its drawbacks, and the method of choice is a combination of suitable methods or a careful choice of the method most suitable for the specific problem considered. In this article we focus on two complimentary manifold methods which answer the questions above in most complete form.

\section{GENERAL FRAMEWORK - COORDINATE FREE SINGULAR PERTURBATIONS}

The geometrically invariant concept of singularly perturbed systems (SPS) of ordinary differential equations is discussed in this section. Locally this concept is equivalent to the standard SPS if a necessary nonlinear transformation of coordinates is known. However this is not the case if one needs the global information about the decomposition discussed above.

\subsection{Coordinate versus Coordinate Free Formulations}

The standard mathematical framework to handle multiscales phenomenon is the standard SPS theory (see e.g. [24, 25] for more detail). In short, it is supposed by the SPS theory that the original variables are already suitable for multiscales analysis. It means that the system is represented in such a way (as a SPS), that the fast relaxation processes is driven by the set of original variables, i.e. fixed set of the original variables are assumed to be "frozen" i.e. change slowly during the fast relaxation.

However, it is clear that by working in the original coordinates one restricts considerably the applicability range of such an analysis. For instance, Fig. (4) shows the system solution trajectories for the benchmark syngas/air system

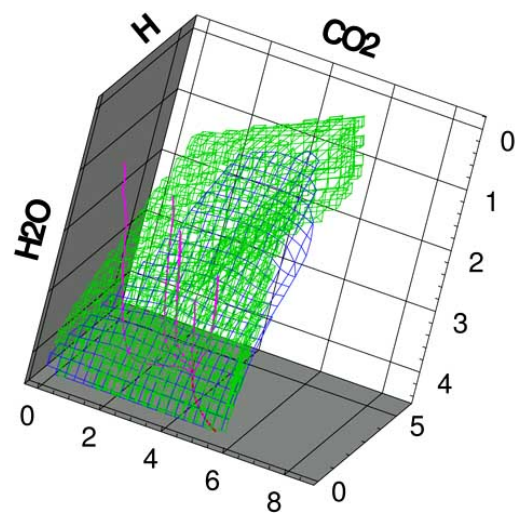

Fig. (4). Results of ILDM analysis of a syngas/air premixed flame shown in projection onto specific mole numbers of several species. Magenta lines are solutions' trajectories, the blue mash is the 2D ILDM, and the green mesh the 3D ILDM. 
where one sees the fast relaxation process can not be captured correctly by original variables even by variables representing major species (left), but nevertheless it is seen that the relaxation follows quite simple linear hyper-planes (magenta lines). Therefore, a coordinate free approach has been developed recently [26, 27]. The main subject of the study is to access the decomposition structure without being restricted to particular coordinate frame. This approach allowed reformulating the problem of model reduction in a general case.

As a result of the investigation an algorithm for identification of the hierarchical decomposition and its suitable representation has been developed (see e.g. [27, 28] for more detail).

\subsection{Global Quasi-Linearisation (GQL)}

The main theoretical questions that algorithm answers automatically are the following:

- Whether a given system can be decomposed linearly or not? The reader should not be confused by the word linear because, it refers to the decomposition only and not to the original system or manifold needed for model reduction.

- What is the system small parameter i.e. a measure of discrepancy in different scales?

- What is the minimal dimension of the decomposed form required for reduction purposes?

- How can the fast linear subspace be found?

By answering the questions above the GQL approach completes the original manifold ILDM method in the following sense: it allows a global analysis of the system hierarchy, while the ILDM concept provides with important local characteristics of (1). According to the GQL method, the coordinate system that represents the original system in an explicit decomposed form can be found by a linear transformation of the original coordinate system according to main guidelines of the ILDM approach [2]. The multi-scale nature of the processes results in a gap between the eigenvalues of the global linear approximation or interpolation of the Right Hand Side (RHS) of (1) given by $T \psi \approx F(\psi)$. It means that there are two groups of eigenvalues that have sufficiently different (by orders of magnitude) characteristic absolute values. Their invariant subspaces $Z_{f}, Z_{s}$ yield corresponding representation by using the Schur decomposition and then by solving subsequently the Sylvester equation [2]:

$$
\begin{aligned}
& T=\left(\begin{array}{ll}
Z_{s} & Z_{f}
\end{array}\right)\left(\begin{array}{cc}
\Lambda_{s} & 0 \\
0 & \Lambda_{f}
\end{array}\right)\left(\begin{array}{c}
\tilde{Z}_{s} \\
\tilde{Z}_{f}
\end{array}\right), \\
& Z \cdot \tilde{Z}=\left(\begin{array}{ll}
Z_{s} & Z_{f}
\end{array}\right)\left(\begin{array}{c}
\tilde{Z}_{s} \\
\tilde{Z}_{f}
\end{array}\right)=I_{n \times n},
\end{aligned}
$$

where $\Lambda_{f}, \Lambda_{s}$ (block matrices) correspond to these groups of eigenvalues. Thus, a manifold describing the slow system dynamics is given as the manifold that annihilates the subprocesses spanned into the fast subspace, i.e. it defines the states where fastest processes are relaxed already:
$M=\left\{\tilde{Z}_{f} \cdot F(\psi)=0\right\}$.

This equation system is an implicit definition of the correlation between the state variables of a reacting flow introduced by fast relaxing thermo-chemical processes. Thus, new coordinates suitable for an explicit decomposition are given by

$$
\left(\begin{array}{l}
U \\
V
\end{array}\right)=\tilde{Z} \psi=\left(\begin{array}{c}
\tilde{Z}_{s} \psi \\
\tilde{Z}_{f} \psi
\end{array}\right),
$$

where $U$ and $V$ correspond to new fast and slow variables respectively. The small system parameter can be estimated by the gap between the smallest eigenvalue of the large group and the largest eigenvalue of the small group of eigenvalues [28].

$\varepsilon=\frac{\max \left(\left|\lambda\left(\Lambda_{s}\right)\right|\right)}{\min \left(\left|\lambda\left(\Lambda_{f}\right)\right|\right)}$.

Now, it is defined globally over the whole domain $\Omega$ in the state space of (1). Additionally, one of the major advantages of the constructed decomposition by the GQL is the fact that in order to decompose the system and find the slow/fast manifolds in the whole domain it is not needed to find the slow manifold itself (tabulate it), but the matrix $\mathrm{T}$ or its invariant subspaces can be used instead (see (5)-(7)).

Other advantages are the following: the reduced system dimension and the small system parameter are available constant; the numerical effort for the evaluation of the manifold equation can be reduced considerably, because the computationally expensive procedure of decomposition into invariant subspaces is performed only once; the analysis of the slow invariant manifold's properties (stable, turning, repulsive etc.) becomes possible due to the constant decomposition (5) and related to the following eigenvalue problem

$\left.\operatorname{Re}\left(\lambda\left(\tilde{Z}_{f} F_{\psi}(\psi) Z_{f}\right)\right)\right|_{\psi \in M}<0$.

This condition means that all points of the manifold are locally attractive to the system trajectory flow. Thus, without proper estimation of the invariant subspace of the fast motions this feature of the manifold cannot be investigated.

The method has been implemented in the ILDM code [29]. In order to verify the method and investigate its applicability limits it has been applied to the n-heptane autoignition problem [30]. 2D GQL slow manifold is presented in Fig. (5), note that the standard 2D ILDM was not found even near the equilibrium point for the studied skeletal mechanism. One sees on Fig. (5) the comparison of the detailed and reduced model solutions in projection to species specific mole numbers.

Although low dimensional slow manifolds exist (see Fig. 5) they do not perform well in the context of ignition problems. However, in this particular case application of the GQL algorithm yields a 14 dimensional slow GQL manifold that can be successfully used to formulate the reduced model and reproduce the delay before the final thermal runaway within several percent of accuracy (see [30] for detailed comparison and analysis). 


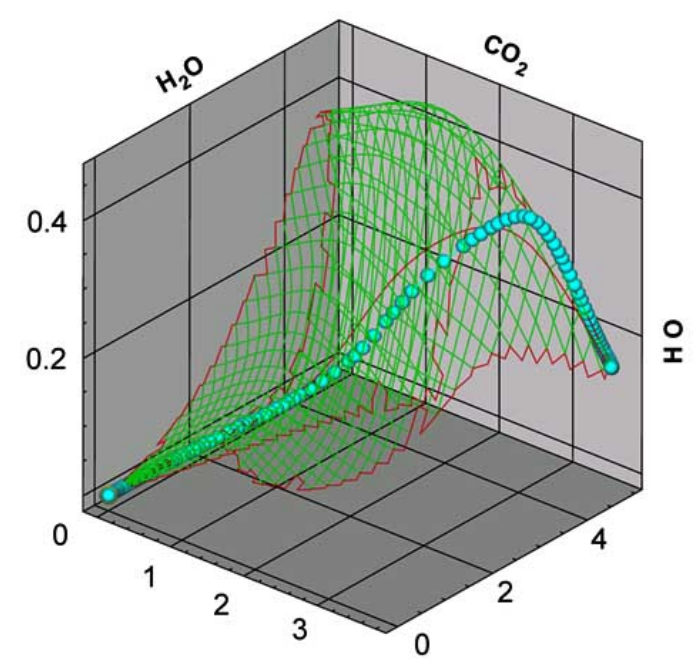

Fig. (5). State space projection onto specific mole numbers with detailed system solution trajectory shown by the red line, 2D GQL slow manifold is the green mesh, cyan line with spheres is the reduced model solution trajectory. n-heptane auto-ignition problem [30].

\subsection{Discussion of the Coordinate Free Framework}

It has to be underlined once more that the discussed method of automatic mechanism reduction and analysis is a realization of the general scheme of an automatic reduction procedure based on the ILDM approach, with the difference that a slow invariant manifold of low dimension and system decomposition is evaluated by the developed GQL procedure instead of the Jacobian matrix. Moreover, the suggested approach follows well-known ideas from the standard ILDM method, which allows us to use developed ILDM codes to implement the approach, but there are some important novelties making it very efficient and attractive for applications.

- the global decomposition is found using invariant subspaces of the GQL matrix in the whole domain of interest of the state space,

- the reduced system dimension is defined by the eigenvalue analysis of the GQL matrix (5),

- the structure of the decomposition remains fixed, such that a correct projection becomes available (7),

- the numerical methods for the evaluation of the manifold equation (6) is simplified considerably, because the computationally expensive procedure of decomposition into invariant subspaces (5) has to be performed only once,

- an estimation of the small system parameter (8) is obtained globally by the suggested approach,

- the analysis of the slow invariant manifold's properties (stable, turning, repulsive etc.) becomes possible due to the global system hierarchy (9).

\section{COUPLING WITH TRANSPORT - DEFINITION OF THE REDUCTION STRATEGY FOR REACTION- DIFFUSION SYSTEM}

This section focuses on the construction of reduced models and implementation schemes for the simulation of combustion processes governed by strongly coupled thermo- chemical and convection/diffusion sub-processes. In order to treat the coupling systematically and account for the influence of transport properties on the reduced model the use of so-called tabulation strategy [31] (in terms of generalized coordinates) and invariant manifolds concept has been proposed in [32, 33]. The resulting REDIM method allows overcoming several problems of the standard ILDM method [12] by implementing a relaxation process which is governed by a multidimensional parabolic partial differential equation system. A problem adapted modification of the REDIM method has been developed as well [34]. A central point of this modification is improving the definition of the reduced model depending on a particular problem configuration. This feature makes the method more problems oriented, and more accurate in predicting the detailed system dynamics (see [34] for more detail). These modifications of the ILDM discussed below improve significantly the performance of the concept and allow extending its area of application.

\subsection{Invariance Condition and the REDIM}

The original system of equations governing the reacting flow of strongly coupled chemical and transport processes can be written $[35,36]$ as

$$
\frac{\partial \psi}{\partial t}=F(\psi)-\vec{v} \operatorname{grad} \psi-\frac{1}{\rho} \operatorname{div}(D \operatorname{grad} \psi) .
$$

Here $D$ is the (n by $\mathrm{n}$ )-dimensional transport matrix, it is a very complicated function of the thermo-kinetic state $\psi$. It is supposed that there is a manifold determining the reduced system dynamics (3), but valid for the system of PDEs (10) as well. By simple substitution and reformulation of (10) on the manifold (3) one obtains

$\psi_{\theta} \frac{\partial \theta}{\partial t}=F(\psi(\theta))-\vec{v} \psi_{\theta} \operatorname{grad} \theta-\frac{1}{\rho} \operatorname{div}\left(D \psi_{\theta} \operatorname{grad} \theta\right)$

Now, the Moore-Penrose pseudo-inverse matrix $\psi_{\theta}^{+}=\left(\psi_{\theta} \psi_{\theta}^{T}\right)^{-1} \psi_{\theta}$ [16] is applied as before to both sides of (11) to see what is important for reduced model in the general case of (10)

$\frac{\partial \theta}{\partial t}=\psi_{\theta}^{+} F(\psi(\theta))-\vec{v} \operatorname{grad} \theta-\psi_{\theta}^{+} \frac{1}{\rho} \operatorname{div}\left(D \psi_{\theta} \operatorname{grad} \theta\right)$.

It is obvious now that both reaction and diffusion influence the reduced model behavior through the manifold's structure (12). Accordingly, in order to obtain a consistent manifold (i.e. reduced model) the influence of both has to be studied and included into the manifold definition.

This situation is illustrated by Fig. (6), where one can see that the system's stationary solution deviates from the ILDM (blue mesh), it looses the attractive properties and even ceases to exist (red curve), while the REDIM (green mesh) captures quite well the stationary system behavior everywhere.

In order to implement the manifold concept efficiently for model reduction and improve the situation with coupling of both terms within the reduced model the geometrical framework of the invariant sets is applied to (10). 


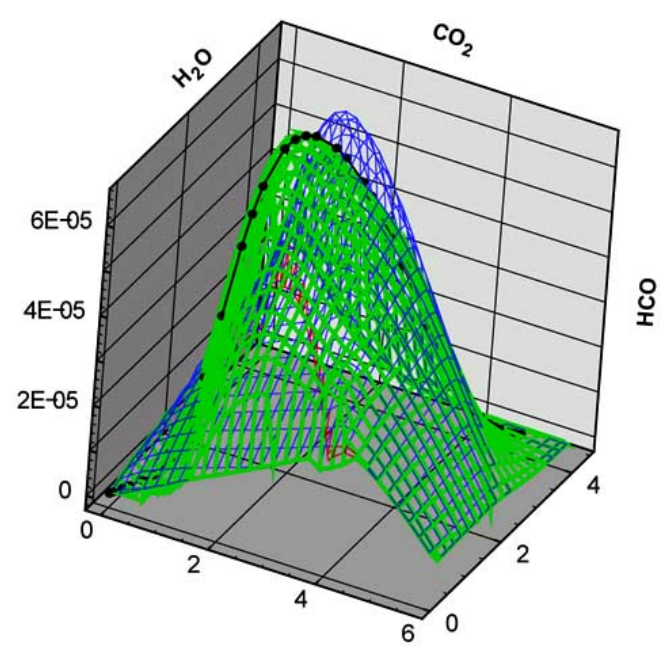

Fig. (6). State space projection onto specific mole numbers with detailed system solution trajectory shown by the black line, 2D ILDM (extended) slow manifold is the blue mesh, 2D REDIM is shown by the green mesh.

An assumption that an invariant slow manifold of low dimension $M(\operatorname{dim} M=m<<n)$ exists in the state space as an intrinsic property of the combustion system yields

$$
\begin{aligned}
& \left(I-\psi_{\theta}\left(\theta_{0}\right) \psi_{\theta}^{+}\left(\theta_{0}\right)\right) . \\
& \cdot\left\{F\left(\psi\left(\theta_{0}\right)\right)-\frac{1}{\rho} \operatorname{div}\left(D \operatorname{grad} \psi\left(\theta_{0}\right)\right)\right\}=0
\end{aligned}
$$

which means the vector field defined by (10) belongs to the tangent subspace of the manifold at any point $\theta_{0}$ on it. The invariance condition (13) is a projection of the vector field onto the normal subspace of the manifold that has to vanish on the slow invariant manifold. An identity matrix $I$ and the projection operator $P_{(T M)^{\perp}}=\left(I-\psi_{\theta} \psi_{\theta}^{+}\right)$onto the normal subspace of $M$ have been used in (13). Invariance is a very important property that guaranties the solution of the overall detailed model will be confined or restricted to the manifold used for model reduction, i.e. only invariant manifolds are appropriate for reduction purposes. Equation (13) is solved by introducing an iterative procedure which is based on a reformulation as a multidimensional parabolic system [12]:

$$
\left\{\begin{array}{l}
\frac{\partial \psi(\theta, t)}{\partial t}=\left(I-\psi_{\theta}(\theta) \psi_{\theta}^{+}(\theta)\right) \cdot \\
\cdot\left[F(\psi(\theta))-\frac{1}{\rho} \operatorname{div}\left(D \psi_{\theta}(\theta) \operatorname{grad} \theta\right)\right] \\
\psi(\theta, 0)=\psi_{I L D M}(\theta)
\end{array}\right.
$$

with initial and boundary conditions given by an extended ILDM manifold $\psi=\psi_{I L D M}(\theta)$ (shown on Fig. (6) by the blue mesh). Obviously, the stationary solution of this system would satisfy exactly the co-called invariance condition (13) and, therefore, approximates the manifold appropriate for the reduced system formulation. The only problem with (14) is the transport term which contains the gradient of the parameter of the manifold, i.e. the dependence of the reduced mani- fold on the spatial variable. This dependence has been studied extensively, where a constant approximation of the parameter's gradient $\operatorname{grad} \theta$ was introduced, showing that the final reduced model is not sensitive to this gradient (see e.g. [12] for more detail of the analysis). Fig. (6) illustrates the results of the implementation of the relaxation method (14) to the flat flame syngas/air combustion system. It can be seen that the REDIM method improves the results considerably.

\subsection{Problem Adapted REDIM}

In the case when accurate and relatively low dimensional models (1D, 2D etc.) are required, the estimate (15) can decrease the accuracy of the REDIM [12]. In order to improve the constant estimate (15) of the parameter gradient an approximation of the reduced system gradient as a function of the parameter itself [34] has been proposed. By an iterative procedure shown below that solve the invariance equation (14) and by a test integration of the resulting reduced model (12) the problem of estimating the gradient can be overcome.

The proposed method, which does not depend on the gradient estimates and, therefore, represents a better way to overcome the problem of dependence on the spatial coordinates, is briefly outlined yet.

First, a constant approximation

$\operatorname{grad} \theta=$ const ,

in (14) is used in order to obtain the first approximation of the slow manifold $\psi^{1}(\theta)$ in (3). In [12] it has been shown that even for the constant approximation it is possible to achieve an appropriate accuracy of the reduced model, especially, if the dimension of the reduced model is high. Second, the reduced model $\psi^{1}(\theta)$ is used for integration to obtain the reduced model's stationary solution $\theta^{1}(x)$ by solving the following reduced system for $\theta=\theta(x, t)$ :

$$
\begin{aligned}
& \frac{\partial \theta(x, t)}{\partial t}=\psi_{\theta}^{1,+} F\left(\psi^{1}(\theta)\right)-\vec{v} \operatorname{grad} \theta- \\
& -\psi_{\theta}^{1,+} \frac{1}{\rho} \operatorname{div}\left(D \psi_{\theta}^{1,+} \operatorname{grad} \theta\right)
\end{aligned}
$$

Third, $\theta^{1}(x)$ is used to find an approximation of the gradient of the manifold's parameter as

$$
\operatorname{grad} \theta=\frac{\partial \theta^{1}(x)}{\partial x}
$$

and the gradient's dependence on $\theta, \operatorname{grad} \theta=f_{1}(\theta)$ is determined. Now, the first step can be repeated with $\operatorname{grad} \theta=f_{1}(\theta)$ instead of constant approximation (15) and so on. One obtains constantly improved with iterations representation of the gradient on the manifold $f_{i}(\theta)$, which can be used in the next iteration to improve the reduced model (manifold) together with $\psi(\theta, 0)=\psi^{i}(\theta)$ as a starting solution in (14). The REDIM method has also been implemented in the codes INSFLA and HOMREA [2, 29]. Fig. (7) illustrates the iterations process of constantly improving manifold representation with the improved reduced model solu- 

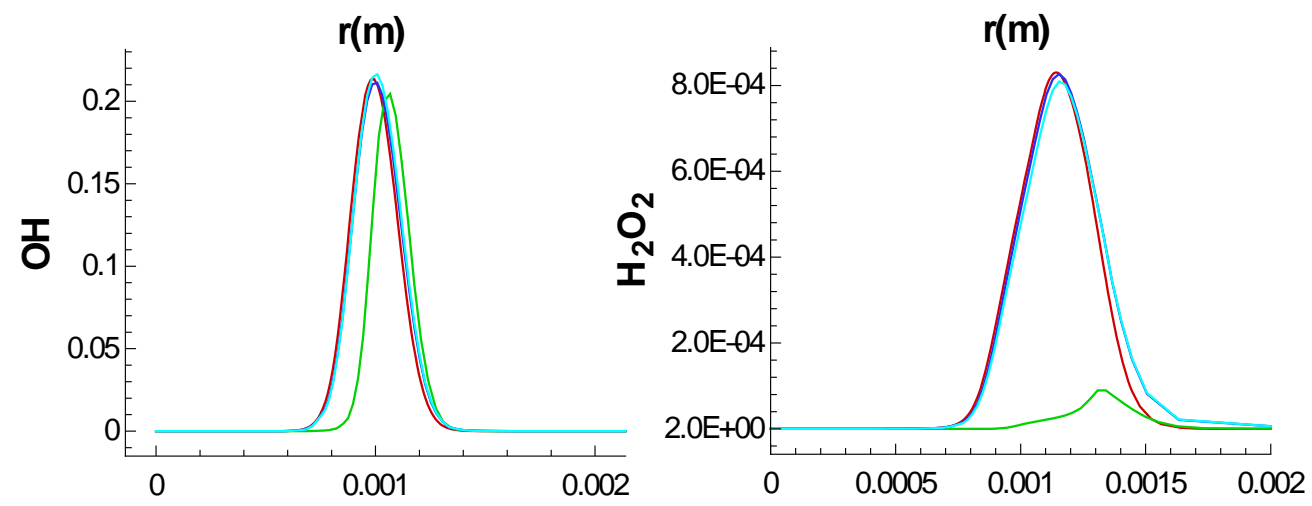

Fig. (7). Syngas/air diffusion flame. $\mathrm{OH}$ - (left) and $\mathrm{H}_{2} \mathrm{O}_{2}$ - specific mole numbers profiles (right). The detailed solution is shown by the red line, the green line represents the result of the first iteration for the constant gradient over the spatial domain; the cyan line is the result of the second iteration and the blue line corresponds to the third one [34].

tion for diffusion flame of the syngas/air system. In this case a very rough initial guess of a constant gradient has been applied [34].

The REDIM includes very important novelties that render it very efficient in realization of the general scheme of automatic reductions. The main features of the REDIM method are

- an approximation for the slow system manifold avoiding the dependence on the spatial coordinates is proposed such that problems with infinite dimensional manifold structures are overcome;

- the approximation is given in an explicit form as a stationary solution of a multidimensional parabolic system;

- a starting or an initial solution of the approximate manifold is constructed using the extended ILDM method. This increases the robustness of the method because it guarantees better convergence and stability of integration of the REDIM equation system.

\section{CONCLUSIONS}

Manifold methods as a suitable framework of model reduction are discussed comprehensively in the present paper. The problem of model reduction is treated in a general formulation by considering first the ODEs system as a simplest model of reacting system. The natural assumption of multiscale phenomena and the decomposition of motions decouple the fast motions/processes and as a result reduce the system's dimension and stiffness of such models making them treatable numerically.

The main feature of the first discussed approach in comparison to other approaches is a global character. It is capable of approximating not only the slow system dynamics, but the fast manifolds as well. It was discussed that the approach can be applied in both analytical and numerical aspects to yield the explicit decomposition of the system exhibiting the hierarchical (multi-scale) behavior. On this basis a robust, efficient and automatic algorithm for kinetic mechanisms analysis and reduction has been proposed [28]. The realization and implementation of the method follow the general scheme of an automatic reduction procedure based on the ILDM approach, the difference - a slow invariant manifold of low dimension and system decomposition is evaluated by the developed GQL procedure instead of local analysis based on the Jacobian matrix.

The second discussed method accounts for strong coupling and influence of the transport effects on the reduced model. The system of governing PDEs is considered and treated successfully within the manifold framework. A representation of manifold in terms of generalized coordinates offers the advantage of a robust and efficient implementation and development of the REDIM method. Finally, an improvement of the REDIM method is outlined. The reduced model is integrated together with the REDIM equation system iteratively that improves parameter's gradient as a function of the parameter (local coordinate of the manifold). The procedure is recursive and allows improving the reduced model step by step which makes the reduced model more problems oriented.

The proposed methods supplied by the tabulation strategy are very efficient tools in a CFD modelling of industrial scale applications because, typically, detailed comprehensive mechanisms are computationally prohibitive for many reacting flows (e.g. turbulent three-dimensional flames). The main goal of future work should be, in our opinion, the application of the developed methods to fully transient combustion processes, where phenomena like extinction and reignition can occur.

\section{ACKNOWLEDGMENTS}

The authors acknowledge gratefully the financial support by Deutsche Forschungsgemeinschaft (DFG) within the SFB 606 and SPP 1276 - MetStöm programs.

\section{NOMENCLATURE}

$n \quad=$ Overall system dimension

$n_{s}=$ Number of chemical species participating in the chemical reaction

$n_{r} \quad=\quad$ Number of elementary reactions of the chemical kinetics mechanism

$\psi=$ Thermo-chemical state space vector

$h=$ Enthalpy

$p \quad=$ Pressure

$w_{i} \quad=$ Species mass fraction

$M_{i} \quad=\quad$ Species molar masses 
$\vec{v} \quad=$ Flow velocity vector field

$D=$ Generalized transport matrix

$F \quad=$ Source term in vector form

$M \quad=$ Manifold describing the reduced model in the state space

$\theta=$ Generalized coordinates used to parameterize the manifold

$\psi_{\theta} \quad=$ Jacobian matrix of the manifold

$\psi_{\theta}^{+}=$Moore-Penrose pseudo-inverse of the Jacobian matrix

$F_{\psi} \quad=\quad$ System Jacobian matrix

$Z$ = Local or global coordinate transformation matrix

$Z_{f} \quad=\quad$ Invariant subspace of the Jacobian matrix corresponding to large and negative eigenvalues

$Z_{s}=$ Invariant subspace of the Jacobian matrix corresponding to small eigenvalues

\section{REFERENCES}

[1] U. Maas and S. B. Pope, "Simplifying chemical kinetics: Intrinsic low-dimensional manifolds in composition space", Combust Flame, vol. 88, pp. 239-264, March 1992.

[2] U. Maas, Automatische Reduktion von Reaktionsmechanismen zur Simulation reaktiver Stroemungen, Habilitation thesis, Universitaet Stuttgart: Stuttgart, Germany, 1993.

[3] C. Rhodes, M. Morari and S. Wiggins, "Identification of low order manifolds: Validating the algorithm of Maas and Pope", Chaos, vol. 9, pp. 108-123, 1999.

[4] I. Goldfarb, V. Gol'dshtein and U. Maas, "Comparative analysis of two asymptotic approaches based on integral manifolds", IMA J. Appl. Math., vol. 69, pp. 353-374, 2004.

[5] S. H. Lam and D. M. Goussis, "The CSP method for simplifying kinetics”, Int. J. Chem. Kinet., vol. 26, pp. 461-486, 1994.

[6] S. Singh, J. M. Powers and S. Paolucci, "On slow manifolds of chemical reaction flows", J. Phys. Chem., vol. 117(4), pp. 14821496, 2002.

[7] S. B. Pope and U. Maas, Special publication, Cornell Report, 1993.

[8] J. A. van Oijen and L.P.H de Goey, "Modelling of premixed counter-flow flames using the Flamelet Generated Manifold Method", Combust Theor. Model., vol. 6, pp. 463-478, 2002.

[9] W.P. Jones and S. Rigopoulos, "Rate-controlled constrained equilibrium: formulation and application to nonpremixed laminar flames", Combust Flame, vol. 142, pp. 223-234, 2004.

[10] D. Lebiedz, "Computing minimal entropy production trajectories: an approach to model reduction in chemical kinetics", J. Chem. Phys., vol. 120(5), pp. 6890-6897, 2004.

[11] U. Maas, Mathematical Modeling of the Coupling of Chemical Kinetics with Flow and Molecular Transport, in Scientific Computing in Chemical Engineering II, Springer, 1999; pp. 26-56.

[12] V. Bykov and U. Maas, "The extension of the ILDM concept to reaction-diffusion manifolds", Combust Theor. Model., vol. 11(6), pp. 839-862, 2007.
[13] N. Peters and B. Rogg, Reduced Kinetics Mechanisms for Application in Combustion Systems, Springer Verlag Berlin Hiedelberg, New York, 1993.

[14] F.A. Williams, Combustion Theory, $2^{\text {nd }}$ ed. Benjamin-Cummings, Menlo Park, California, 1985.

[15] J. Warnatz, U. Maas and R.W. Dibble, Combustion, $4^{\text {th }}$ ed. Springer-Verlag, New York, 2006.

[16] K.J. Laidler, Chemical Kinetics, $3^{\text {rd }}$ ed. Benjamin-Cummings, CA, 1997.

[17] G.H. Golub and C. F. van Loan, Matrix Computation, The Hopkins University Press: Baltimore, London, 1989.

[18] M. Bodenstein and S.C. Lind, "Geschwindigkeit der Bildung des Bromwasserstoffs aus seinen Elementen“, Z. Phys. Chem., vol. 27, pp. 168-175, 1906.

[19] J. R. Bowen, A. Acrivos, and A. K. Oppenheim, "Singular perturbation refinement to Quasi-Steady State Approximation in chemical kinetics“, Chem. Eng. Sci., vol. 18, pp. 177-188, 1963.

[20] U. Maas und D. Thévenin, "Correlation analysis of direct numerical simulation data of turbulent non-premixed flames“, Proc. Combust. Inst., vol. 27, pp. 1183-1189, 1998.

[21] T. Blasenbrey und U. Maas, "ILDMs of higher hydrocarbons and the hierarchy of chemical kinetics“, Proc. Combust. Inst., vol. 28, pp. 1623-1630, 2000.

[22] Z. Ren, S. B. Pope, A. Vladimirsky and J. M. Guckenheimer, "Application of the ICE-PIC method for the dimension reduction of chemical kinetics coupled with transport", Proc. Combust. Inst., vol. 31, pp. 473-481, 2006.

[23] M. A. Singer, S. B. Pope and H. N. Najm, "Operator-splitting with ISAT to model reacting flow with detailed chemistry", Combust Theor. Model., vol. 10(2), pp. 199-217, 2006.

[24] N. Fenichel, "Geometric singular perturbation theory for ordinary differential equations", J. Differ. Equ., vol. 31, pp. 53-98, 1979.

[25] V. Gol'dshtein and V. Sobolev, Amer. Math. Soc., Translations, ed.: S.G. Gindikin, s. 2, 153 (1992), pp. 73-92.

[26] V. Bykov, I. Goldfarb and V. Gol'dshtein, "Novel numerical decomposition approaches for multiscale combustion and kinetic models", J. Phys: Conf Ser, vol. 22, pp. 1-29, 2005.

[27] V. Bykov, I. Goldfarb and V. Gol'dshtein, "Singularly perturbed vector fields", J. Phys. Conf. Ser., vol. 55, pp. 22-44, 2006.

[28] V. Bykov, V. Gol'dshtein and U. Maas, "Simple global reduction technique based on decomposition approach", Combust. Theor. Model., vol. 12(2), pp. 389-405, 2008.

[29] U. Maas and J. Warnatz, "Ignition processes in hydrogen-oxygen mixtures", Combust. Flame, vol. 74, pp. 53-69, 1988.

[30] V. Bykov and U. Maas, "Investigation of the hierarchical structure of kinetic models in ignition problems", Z. Phys. Chem., vol. 223(4-5), pp. 461-479, 2009.

[31] J. Bauer, V. Bykov and U. Maas, "Implementation of ILDMs based on a representation in generalized coordinates", in Proc. Eu. Con. Comp. Fluid Dyn, Egmond aan Zee, The Netherlands, 2006.

[32] A. N. Gorban and I. V. Karlin, "Method of invariant manifold for chemical kinetics", Chem. Eng. Sci., vol. 58, pp. 4751-4768, 2003.

[33] A. N. Gorban, I. V. Karlin and A. Yu. Zinovyev, "Constructive methods of invariant manifolds for kinetic problems", Phys. Rep., vol. 396(4-6), pp. 197-403, 2004.

[34] V. Bykov and U. Maas, "Problem adapted reduced models based on Reaction-Diffusion Manifolds (ReDiMs)", Proc. Combust. Inst., vol. 32(1), pp. 561-568, 2008.

[35] J.O. Hirschfelder and C.F. Curtiss, Molecular Theory of Gases and Liquids, Wiley: New York, 1964.

[36] U. Maas, und U. Nowak, ZIB Preprint (1996), pp. 96-59.

(C) Bykov and Maas; Licensee Bentham Open.

This is an open access article licensed under the terms of the Creative Commons Attribution Non-Commercial License (http://creativecommons.org/licenses/by-nc/3.0/) which permits unrestricted, non-commercial use, distribution and reproduction in any medium, provided the work is properly cited. 